\title{
Des espaces de discussion innovants au service de la biologie médicale
}

BioTribune : Quelles sont les fonctionnalités de ces nouveaux forums?

Nicolas Lacroix : Nous avons axé le développement de ces nouveaux forums sur l'échange et

l'enrichissement des connaissances. Dorénavant, les utilisateurs pourront mettre en commun images, fichiers et liens accompagnés d'un commentaire, et les partager avec tous les membres de l'espace Professionnel de BioTribune ou seulement avec les membres de leur groupe (dans le cas des forums privés). De plus, lors de la rédaction d'une contribution, l'utilisateur peut d'un simple clic faire référence à un ou plusieurs de ces éléments partagés et envoyer son intervention sur plusieurs forums.

A tout moment, un utilisateur peut accéder à ses informations personnelles en cliquant sur "mon profil". II peut ainsi modifier sa signature, les alertes depuis chaque forum l'avertissant de nouvelles contributions ou de réponses à ses messages.

BT : Quelles sont les avantages de ces forums par rapport à une liste de diffusion classique?

NL : Ils sont multiples et je n'aborderai ici que les principaux points forts. En plus de reprendre les fonctionnalités classiques d'une liste de diffusion, ces forums offrent plus de sécurité. En effet, tout utilisateur désirant envoyer un message au groupe auquel il appartient doit passer par les forums et s'identifier. De plus, tout message est envoyé en copie cachée, c'est-à-dire que les adresses électroniques des membres du groupe ne sont pas visibles, évitant ainsi leur diffusion incontrôlée. Afin de réduire les

\author{
Questions à Nicolas Lacroix, Directeur Technique de \\ BioTribune, à l'occasion du lancement des nouveaux \\ forums ouverts sur BioTribune.com.
}

risques de diffusion de virus informatiques, le partage de fichier et de photo est effectué sur le serveur de BioTribune ; un utilisateur ne peut donc ni envoyer ni recevoir de fichier contaminé à partir de ces forums. Par ailleurs, ce système donne la liberté à l'utilisateur de télécharger les fichiers qu'il souhaite, sans bloquer sa messagerie. Enfin, un utilisateur peut très simplement "cross-poster" un message, c'est-à-dire qu'il peut envoyer une même contribution sur plusieurs forums de spécialité, simultanément, sans avoir à la réécrire.

BT : Qu'en est-il des forums d'experts et des forums privés ? NL : Ces forums correspondent à un besoin qui émane des biologistes. II manquait un lieu où solliciter de manière simple l'opinion d'experts sur des résultats d'analyses donnant lieu à discussion. Ces forums, organisés par spécialités clinico-biologiques, (voir le forum Thyroilde qui inaugure l'ouverture d'une série de forums de spécialité) permettent aux biologistes de poser leurs questions en ligne à des groupes d'experts, d'y joindre un document et d'obtenir les réponses directement dans leur boîte aux lettres électroniques. Le fil de discussion reste disponible sur le forum pour les autres biologistes, enrichissant ainsi une base de connaissance commune.

Les forums privés, ouverts sur demande, permettent de restreindre l'accès à un groupe d'utilisateurs authentifiés.

\begin{abstract}
Chaque groupe peut avoir sa propre identité visuelle (couleur, logo, etc.) et est géré par un ou plusieurs administrateurs.

BioTribune met à la disposition de chaque groupe une interface d'administration permettant de modérer les interventions et les éléments partagès du forum, d'ajouter et de supprimer des utilisateurs, etc.

BT : Et demain?

NL : Nous prévoyons dans un premier temps d'étendre les fonctionnalités de personnalisation du site BioTribune, notamment en permettant aux utilisateurs de gérer leur propre liste de diffusion, c'est à dire leur carnet d'adresse électronique professionnel. Ainsi, ils pourront envoyer les articles qui les intéressent ou inviter des contacts privilégiés à participer à des discussions.
\end{abstract}

\section{BioTribune} générateur de services et d'outils d'échange

\section{Annonces}

Tous les jours de nouvelles annonces en ligne

- Emplois, Remplacements, Stages

- Laboratoires: cessions, acquisitions associations, matériel d'occasion

\section{Agendas}

Ne rater pas un évènement !!!

plus de 450 êvènements rêpertoriés dans nos agendas

Recherches multi-critères : par spécialité, par région ou à l'international, par date de disponibilité. 